\title{
Ricerche geomagnetiche sopra un supposto diapiro
}

\author{
O. Batteidi - A. Francaviglia - M. Giorgi - F. Molina
}

Ricevuto il 26 Luglio 1962

Nella prima edizione della Carta Geologica della Sicilia, e precisamente nel foglio 268 "Caltanissetta ", nella tavoletta "Xirbi " (268 IV SO), poco a Nord della stazione ferroviaria di Caltanissetta Xirbi, allora denominata $\mathrm{S}$. Caterina Xirbi, è segnato uno spuntone di calcare riferito all'Eocene superiore, formante la collinetta di Cozzo dei Disi, attraversato da una intrusione di roccia basica. Questo spuntone è stato descritto da Baldacei $\left.{ }^{(}\right)$come formato da calcare nummulitico e marne a fucoidi riferibile all'Eocene superiore, "attraversato da un piccolo dicco di basalto, ormai quasi distrutto, ma la cui presenza si riconosce bene per l'alterazione prodotta nei calcari stessi e per l'abbondanza di zeoliti che si trovano sparse in quella località ".

Occorre ricordare che, secondo Baldacci, nella zona considerata esiste una lacuna stratigrafica fra l'Eocene superiore e il Miocene medio. Egli, infatti, dice che i terreni fondamentali fra S. Caterina Villarmosa e Caltanissetta sono costituiti da argille mioceniche con strati di sabbie più o meno cementate, che a volte diventano predominanti; $(\cdot i$ informa, inoltre, dell'esistenza di alcune faglie, con direzione predominante NESo, le quali mettono in vista i terreni inferiori, rappresentati da larghe zone di argille scagliose dell'Eocene medio.

Una delle faglie più importanti è quella che ha inizio a sud presso la Mass. Chiapperia, si dirige verso la C.da Garistoppa, passando per l'anzidetta collina di Cozzo dei Disi. Poichè Baldacei ricorda anche dei calcari a rudiste presso la Mass. Pescazzi, ubicata lungo la faglia, e degli scisti marnosi bruni riferiti all'Eocene medio-inferiore, risulta evidente che egli ritiene che nella zona esista una serie stratigrafica normale e continua dal Cretaceo all'Eocene superiore, alla quale seguono gli strati miocenici separati dalla lacuna stratigrafica alla quale abbiamo accennato. In conseguenza, i calcari della collinetta di Cozzo dei Disi, al pari di quelli 
non lontani della Mass. Chiapperia e del M. Marcassita, si trovano in serie regolare e debbono essere considerati in posto.

Successivamente Behrmann () ha pubblicato i risultati di un suo rilevamento geologico della Sicilia centro-meridionale; anche egli riporta nella sua carta l'affioramento del calcare di Cozzo dei Disi attraversato dal dicco di basalto. Benchè questo $A$. abbia considerato molte delle argille scagliose di Baldacei come argille venute in posto per dei particolari fenomeni di diapirismo e abbia considerato tutti gli spuntoni di roce calcarce emergenti dalle argille circostanti come dei microdiapiri, tuttavia considera come diapiri soltanto gli spuntoni di calcari mesozoici e non quelli eocenici, che perciò debbono essere considerati come rocce in posto.

Nel 1948 Floridia $\left(^{3}\right)$ ha pubblicato una nota sopra un preteso fenomeno diapirico nei dintorni di Leonforte (Enna), descrivendo un affioramento di un calcare triassico, attraversato da un dicco di basalto.

Secondo Beneo ('), invece, si tratta di uno "spuntone diabasico di discreta mole, sopportante una placea di calcari scuri a lastre con intercalari di argilla nera, forse del Trias. Fra i calcari e il diabase esiste un'aureola di contatto rappresentata da una piccola fascia di roccia silicea stratiforme che potrebbe essere cornubianite. Intorno allo spuntone si vedono dei banchi di arenarie saccaroidi, di tipo oligocenico, che rappresentano i resti di una catena smembrata i cui elementi sono letteralmente piantati nelle argille circostanti che costituiscono la formazione più rappresentata ". Beneo aggiunge di aver visto una cosa analoga nei pressi di Lercara Friddi (Palermo), e ritiene che tutti i «microdiapiri " di Behrmann debbano essere considerati come schegge più o meno grandi di autoctono siciliano o extra siciliano inglobate e trascinate dalla massa di argille scagliose considerate come facenti parte di una grande coltre di ricoprimento.

Signorini $\left(^{5}\right)$, che ha rilevato la tavoletta "Calascibetta " (268 I NO) nella quale si trova il supposto diapiro, lo considera come un esotico inglobato nella colata gravitativa delle argille scagliose.

Francaviglia $\left(^{6}\right)$ ha considerato come "esotici " tutti i lembi di rocce rigide, ad esclusione di quelle della serie gessoso-solfifera e delle arenarie associate alle argille del Tortoniano, che nel foglio di Caltanissetta sembrano emergere dalla massa argillosa. Infatti, nei campioni prelevati al di sotto dei calcari marnosi a fucoidi del M. Marcassita, nella tavoletta "Imera " (268 IV SE), e di tutti gli altri spuntoni analoghi che formano come una ghirlanda fra il sudetto monte e le masse calcaree della sta- 
zione di Villarosa, è stata riscontrata una microfauna di foraminiferi riferibile senza dubbio al Tortoniano.

In particolare, alla roccia basica di Cozzo dei Disi sono associati dei tufi pieni zeppi di piccole concrezioni di calcite fibrosa, the sono state scambiate per zeoliti.

Avendo considerato come esotici i calcari di Cozzo dei Disi e del microdiapiro di Leonforte, ne consegue che i dicchi intrusi in essi non possono essere radicati, se non ammettendo un vulcanismo post Tortoniano, del quale non si hanno tracce in alcun posto della Sicilia centro-meridionale.

La questione aveva una grande importanza, perchè se le masse calcaree in parola erano degli esotici, bisognava ammettere la alloctonia delle argille scagliose che la inglobano, come ha sostenuto Beneo, il che si ripercuotera nella interpretazione della tettonica regionale, e rendeva necessaria la formulazione di nuove ipotesi di lavoro nella ricerca degli idrocarburi e degli zolfi stessi.

Per avere una conferma della giacitura non radicata dei dicchi in questione, il Servizio Geologico d'Italia, d'accordo con l'Ente Zolfi Italiani, ha dato l'incarico all'Istituto Nazionale di Geofisica di eseguire un rilevamento magnetico nelle due zone, inteso ad accertare se i due diechi si estendessero o no in profondità.

La questione è stata affrontata determinando l'estensione orizzontale delle anomalie magnetiche; infatti, se l'affioramento di roccia basica è costituito da una semplice massa isolata, le anomalie saranno limitate ad una ristretta area attorno alla massa stessa, mentre se essa fa parte di una massa magnetica molto più estesa e profonda, esse saranno sensibili in una regione molto più vasta.

Il rilevamento è stato effettuato nel giugno 1955 mediante due bilance magnetiche Askania Gf6 per la componente verticale, e completato nel maggio 1960 mediante una bilancia Ruska.

Le caratteristiche degli strumenti erano: per la bilancia Askania n. 509998, costante di scala $10,0 \%$ div., coefficiente di temperatura - 0,1 $\mathrm{\gamma} /$ grado; per la bilancia Askania n. 509999, coefficiente di temperatura $+1,6 \gamma /$ grado, costante di scala $11,4 \gamma /$ dir.; per la bilancia Ruska, costante di scala $9,8 \gamma /$ div.

I coefficienti di temperatura sono stati determinati durante la campagna stessa mediante la ripetizione di stazioni fisse a temperatura diversa; le differenze di temperatura, dovute alla escursione diurna, raggiungevano $\mathrm{i} 10^{\circ}$.

Per individuare il " campo normale ", ci siamo serviti di una stazione sicuramente non anomala, e precisamente della stazione fondamentale 
dell'Istituto Geografico Militare n $1294\left({ }^{7}\right)$, situata in contrada Misteci, a Sud di Caltanissetta, stazione che faceva parte anche della rete dell'Istituto Nazionale di Geofisica nel rilevamento della Sicilia centrosettentrionale del $1952\left(^{8}\right)$. La distanza di tale stazione da Cozzo dei I)isi è di circa $10 \mathrm{~km}$ verso Sud; si è dovuto perciò determinare la correzione per la latitudine.

Sono stati all'nopo eseguiti due lunghi profili in direzione N-S: il primo fra Villarosa e Riesi, che nella parte più settentrionale si trova alla stessa latitudine di Cozzo dei Disi e a circa $10 \mathrm{~km}$ verso Est, nonchè poco a Sud del diceo di Leonforte; il secondo dall'incrocio delle strade statali 121 e 122 bis, circa $10 \mathrm{~km}$ a Nord di Caltanissetta (Barriera Noce), fino alla stazione fondamentale di contrada Misteci, circa $6 \mathrm{~km}$ a Sud della città, profilo che attraversa la zona di Cozzo dei Disi.

Sono stati ottenuti i seguenti valori del gradiente di latitudine della componente verticale: per il primo profilo $+7,7 \gamma / \mathrm{km}$, per il secondo $+6,4 \gamma / \mathrm{km}$; tra le due stazioni più settentrionali del primo profilo, la cui latitudine media è uguale a quella della zona oggetto di studio, il gradiente è risultato di $+6,7 \gamma / \mathrm{km}$, in ottimo accordo con il valore del secondo profilo. Nei computi abbiamo scelto quest'ultimo valore, in quanto adatto per la latitudine e non affetto da eventuali influenze delle anomalie da rilevare. La correzione per la variazione diurna della componente verticale del campo magnetico terrestre è stata apportata sulla scorta delle registrazioni magnetiche dell'Osservatorio di Gibilmanna, presso Cefalì, dell'Istituto Nazionale di Geofisica.

La precisione delle singole misure, dedotta dalle differenze dei valori nelle stazioni ripetute ̀े risultata di $\pm 3 \gamma$.

Sulla collina dell'affioramento di Cozzo dei Disi e nella zona immediatamente circostante sono state eseguite un centinaio di stazioni, di cui 73 distribuite in sei profili attraverso l'affioramento stesso, distanziate in ciascun profilo di circa $10 \mathrm{~m}$ l'una dall'altra. Nella Fig. 1 sono rappresentate l'altimetria della zona, le stazioni eseguite con i valori delle anomalie rispetto alla stazione fondamentale di contrada Misteci, e le curve isoanomale, costruite con l'approssimazione consentita dalla distribuzione delle stazioni. La figura mostra una rapida diminuzione delle anomalie fino a valori quasi normali già a distanza dell'ordine dei 50 o 60 metri dall'affioramento.

Nella Fig. 2 sono riportate le stazioni eseguite a maggiore distanza dall'affioramento, il quale è rappresentato dalla zona tratteggiata; i valori di queste stazioni coincidono, entro una diecina di gamma, con i valori del campo normale. 
o. BATTELLT - A. FRANCAVIGLiA - M. GIORgI - F. MOLINA

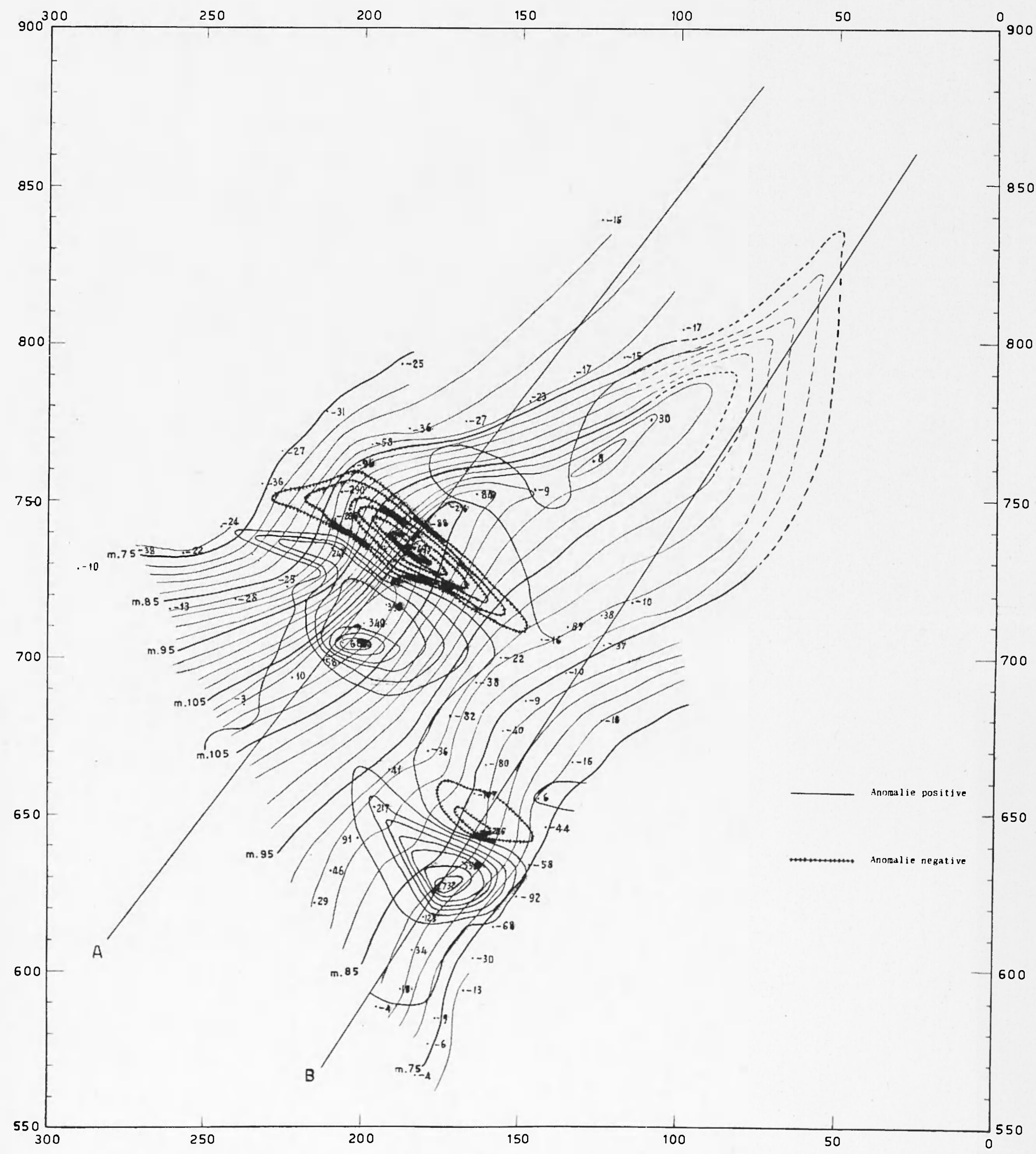

Fig. 1 - Le coordinate espresse in metri sono riferite ad una stazione ubicata vicino al $\mathrm{km} 11$ della strada Caltanissetta-S. Caterina Villarmosa. 
Le isoanomale della Fig. 1 mostrano l'esistenza di due sistemi magnetici distinti. Sono stati ricostruiti due profili pressochè rettilinei in

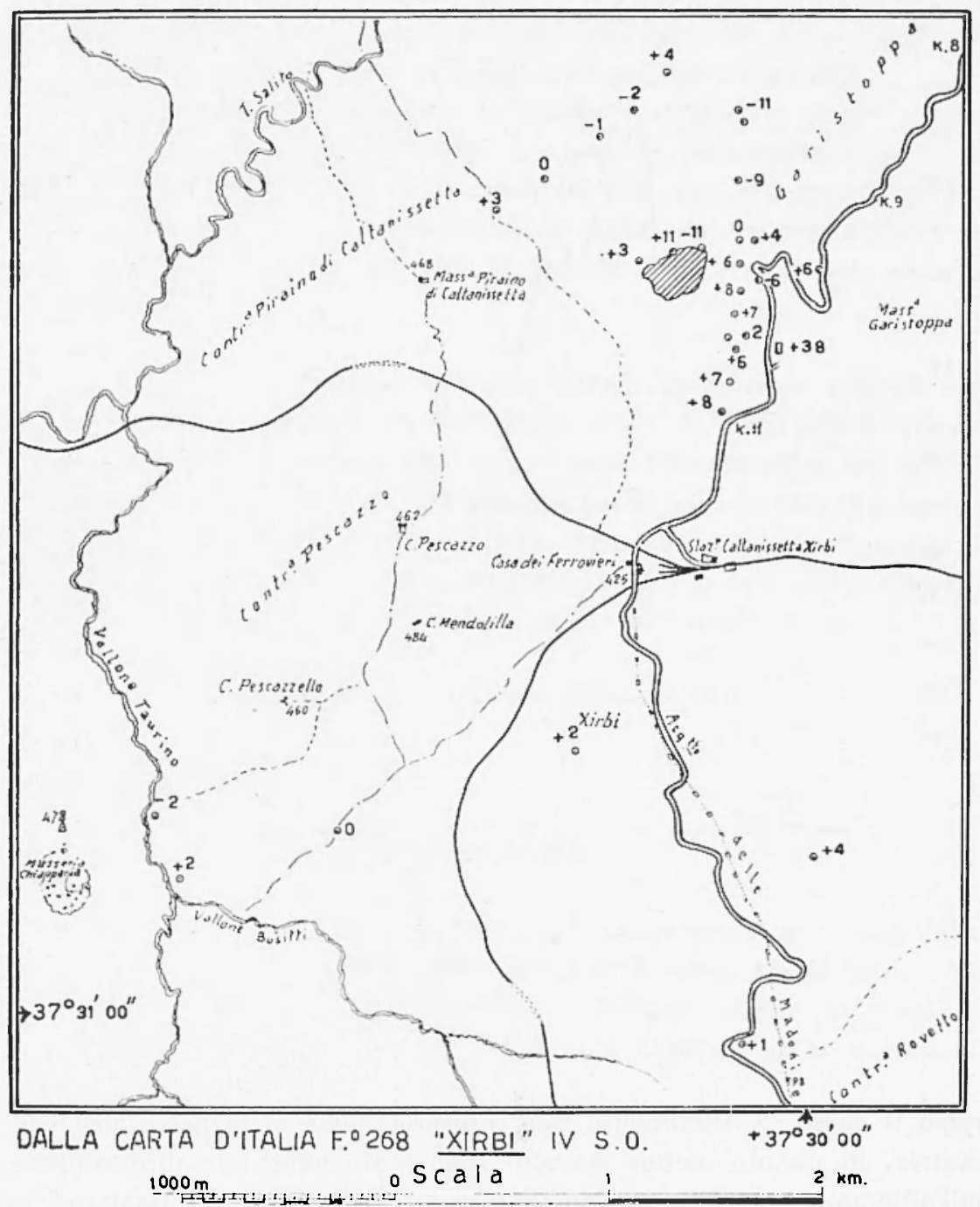

Fig. 2

direzione SO-NE attraversanti i sistemi di anomalie e indicati in figura 1 dalle rette $A$ e $B$. I valori delle anomalie su questi due profili sono riportati nella Fig. 3; le due curve possono esser interpretate come dovute: per il profilo $A$ ad un dipolo orizzontale avente polarizzazione 
coerente con l'attuale campo magnetico terrestre, per il profilo B ad un dipolo nel medesimo verso del precedente, ma inclinato di circa $20^{\circ}$

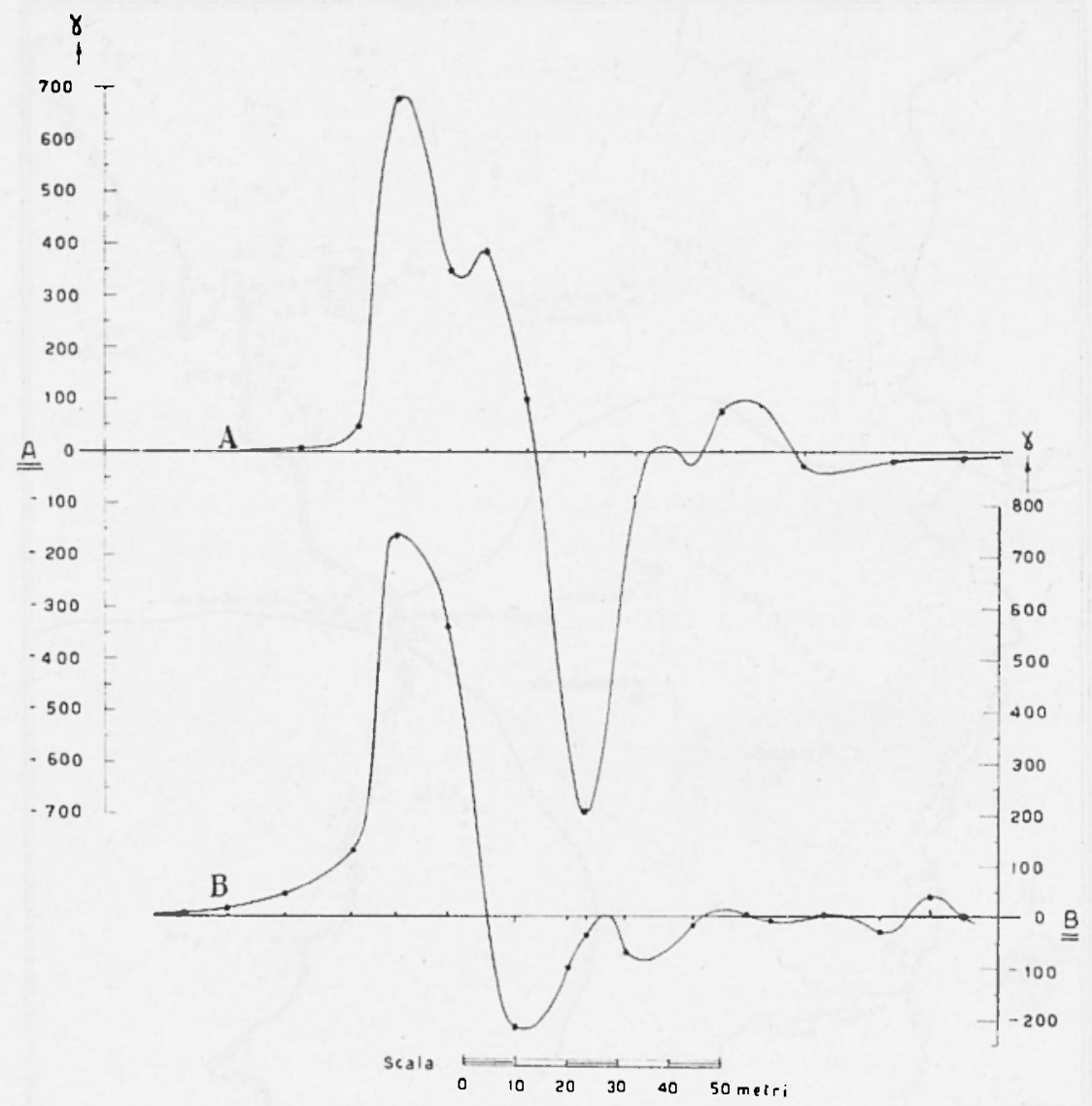

Fig. 3

verso il basso. Naturalmente tale interpretazione è da accogliersi con cautela, in quanto alcune stazioni sono state effettuate direttamente sull'affioramento, e di conseguenza $\mathrm{i}$ valori delle anomalie risultano in esse esaltate.

I risultati suddetti mostrano che anomalie magnetiche sensibili si hanno soltanto nelle immediate vicinanze dell'affioramento; nella regione circostante, fino a distanza di vari chilometri, non sono state rilevate anomalie apprezzabili; in altre parole, il campo, tranne che nella ristretta zona dell'affioramento, appare "normale». 
Questo risultato ci porta a ritenere improbabile l'esistenza in posto di una massa magnetica profonda ed estesa della quale l'affioramento faccia parte. Per conseguenza, la bancata di calcare nella quale è intrusa la roccia basica non può avere radici profonde, e perciò deve essere considerata come una scheggia inglobata nelle argille sottostanti.

Sul presunto "microdiapiro " di Leonforte sono stati eseguiti alcuni profili a titolo orientativo. Siccome le anomalie riscontrate avevano le stesse caratteristiche di quelle di Cozzo dei Disi, non si è creduto necessario procedere ad un rilevamento di dettaglio. Anche per questo caso si ritengono applicabili le conclusioni dedotte dallo studio della zona di Cozzo dei Disi.

Si ringrazia il Servizio Geologico d'Italia per avere suggerito il presente studio e fornito le diverse ipotesi di lavoro, l'Ente Zolfi Italiani per il contributo finanziario che ha permesso l'effettuazione del rilevamento magnetico, e il Rev. Dr. Di Martino per il prezioso aiuto prestatoci durante la prima parte del rilevamento fornendoci tempestivamente le registrazioni magnetiche dell'Osservatorio di Gibilmanna a lui affidato, e per la collaborazione in campagna durante la seconda parte.

Roma, Istituto Nazionalo di Geofisica, Giugno 1962.

\section{RIASSUNTO}

Gli AA. riferiscono sopra un rilevamento magnetico di una zona della Sicilia centrale, inteso a stabilire se un dicco di roccia basica intruso in una bancata calcarea affiorante fra masse argillose potesse far parte di una massa magnetica profonda ed estesa. Il risultato porta a ritenere improbabile questa ipotesi.

\section{$S U M M A R Y$}

The AA. report on a magnetic survey of an area of Central Sinily, meant to establish whether a dike of basic rock inserted in a calcareous layer outcropping among masses of clay, may be part of a deep and wide magnetic mass. The result makes this hypothesis seem improbable. 


\section{BIBLIOGRAFIA}

(1) BaldacCi L., Descrizione geologica dell'Isola di Sicilia. Mem. deser. Carta Geologica d'Italia, v. 1, (Roma, 1886).

(2) Benrmany R. B., Appunti sulla geologia della Sicilia centro-meridionale. Rapporto al Min. Corporazioni, Vacuum Oil Co. in deposito presso il Serv. Geol. d'It., (Roma, 1938).

(3) Flokidia G. B., Di un interessante fenomeno diapirico nei dintorni di Leonforte (Enna). "Giorn. Sc. Nat. Ec. ", 45, sez. 1, (Palermo, 1948).

(') Beneo E., Sul "Microdiapiro" di Leonforte e su quelli a Nord di Gela (Sicilia centrale e meridionale). Rend. Acc. Lincei, el. Sc. Fis. Mat. Nat., s. VIII, 7, (Roma, 1949).

(5) Signorini R., Note descrittive del rilevamento geologico delle tavolette "Villarosa", "Calascibetta" e "Leonforte ". "Boll. Ser. Geol. d'It. ", LXXVII, f. 2, 3, (Roma, 1955).

(6) Francaviglia A., Relazione sul rilevamento delle tavolette "Xirbi" $\mathrm{e}$ "S. Caterina Villarmosa". Relazione inedita all'E.Z.I., (Roma, 1953).

(') Morelli C., La rete geofisica e geodetica in Italia nel suo stato atluale $e$ nei suoi rapporti con la struttura geologica superficiale e profonda. Pubblicazione Ist. Naz. Geof. 121, (Trieste, 1946).

$\left.{ }^{8}\right)$ Giorgi M., Medi E., Molina F., Rilievo magnetico della Sicilia centrosettentrionale. "Annali di Geofisica ", VII, 4, (Roma, 1954). 